\title{
Halogen Bonding and Cooperative Effects in Chlorine Clathrate: Ab Initio Periodic Study Supporting Information
}

\author{
Cristina Cuautli and Ramón Hernandez-Lamoneda* \\ Centro de Investigaciones Químicas CIQ-IICBA, Universidad Autónoma del Estado deMorelos, Av. \\ Universidad 1001, Cuernavaca 62209, Morelos, México \\ E-mail:ramon@uaem.mx
}



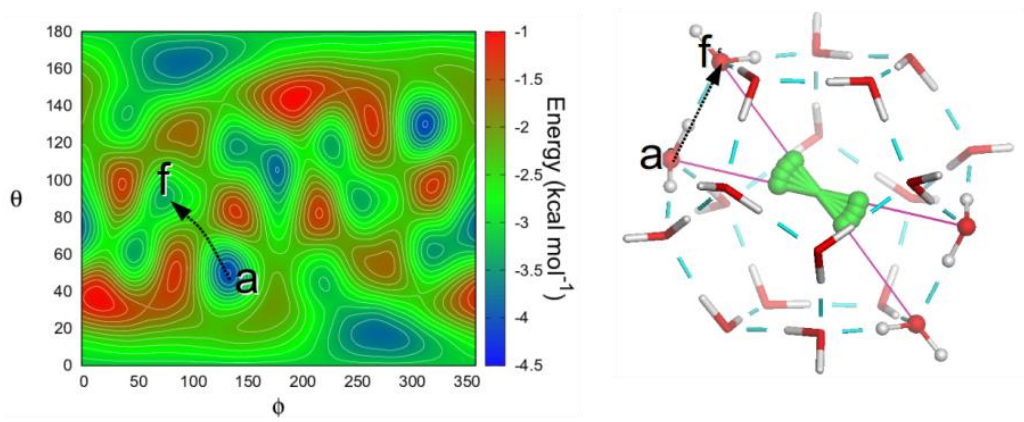

Figure S 1. Minimum energy path trough the stationary points a-f shown in the PES (left) and in the atomic structure(right) for $\mathrm{Cl}_{2} @ 5^{12}$. The water molecules out of the cage are not shown for simplicity.
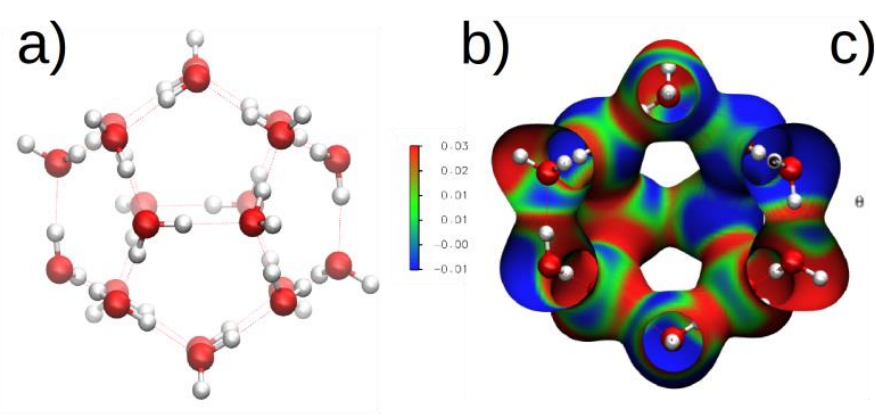

C)

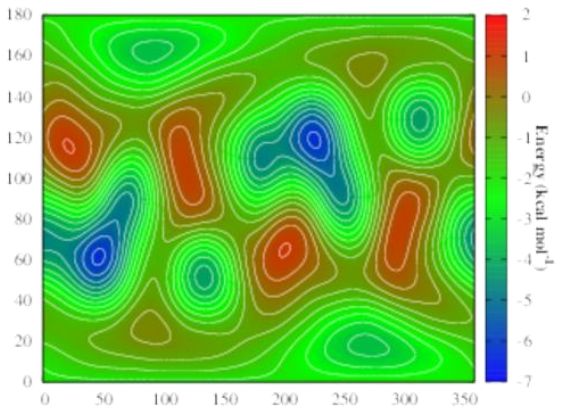

Figure S 2. Atomic structure, electrostatic potential and PES of the $5^{12}$ cage extracted from the crystal. 


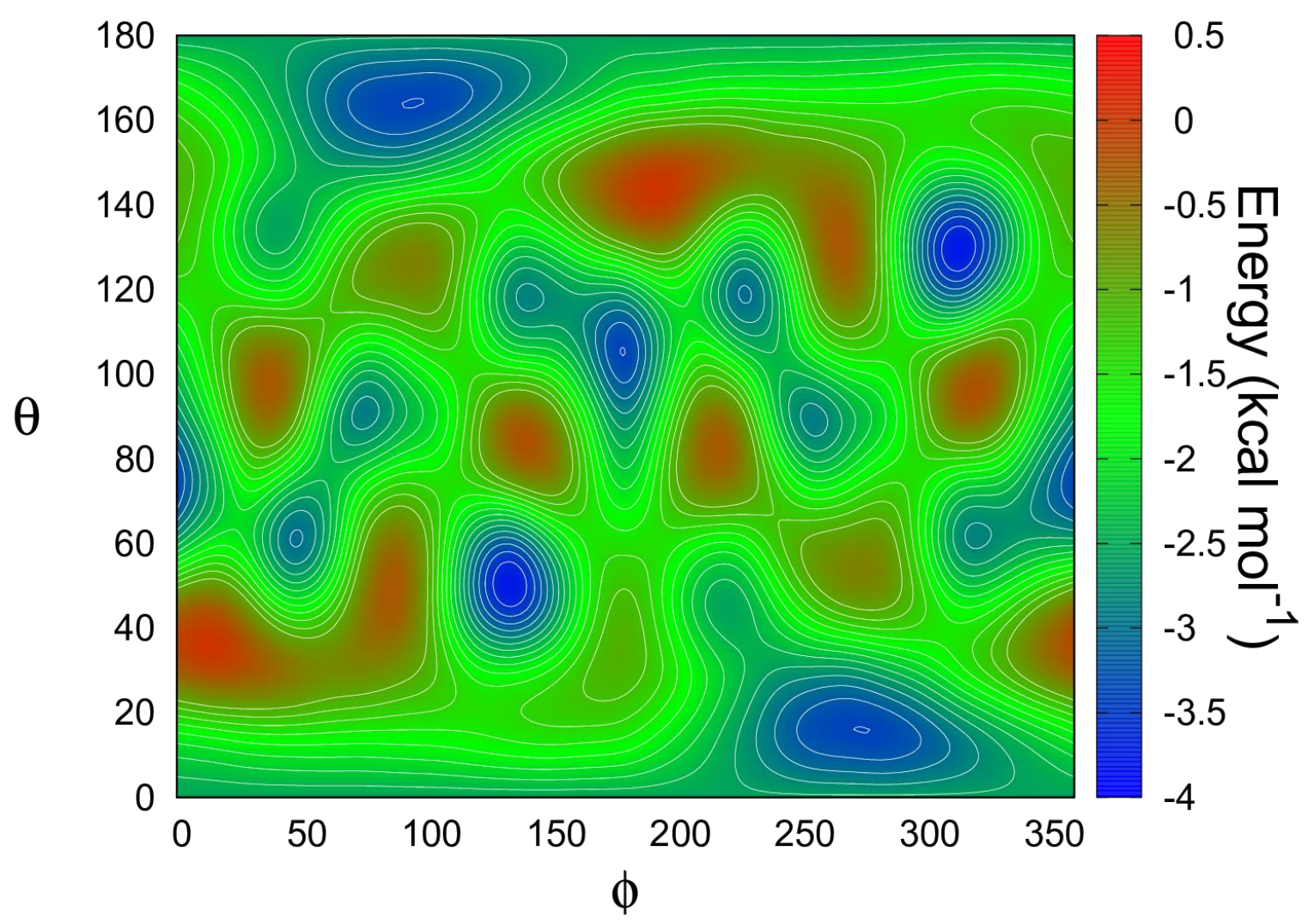

Figure S 3 Angular dependence of the PES for $\mathrm{Cl}_{2} @ 5^{12}$ with the $\mathrm{Cl}-\mathrm{Cl}$ distance $=2.105 \AA$ 